\title{
Attempted suicide in South Asian women
}

\section{Dinesh Bhugra \& Manisha Desai}

Humans have attempted and successfully committed suicide since time immemorial. The reasons for killing or harming oneself vary with cultures and societies and in urban or rural settings.

In ancient European cultures, suicide was common, with women using hanging and men using various tools for deliberate self-harm. Ancient Hindu texts allowed individuals to kill themselves, although in later Upanishadic periods suicide was generally condemned, but it was acceptable for holy men, especially if they had reached the stage in their life where they had gained insight into life's problems. Suicide was permitted on religious grounds, as death was seen as the beginning of another life. Somasundaram et al (1989) report that the great 11th-century Tamil classic Purananuruan anthology includes references to self-immolation by a widow on the death of her husband. There is also mention of suicide by starvation or fasting. These authors conclude that, irrespective of race, religion, culture and location of the civilisation, there was almost identical motivation for suicidal behaviour.

Parasuicide, attempted suicide or deliberate selfharm may be suicidal gestures, manipulative attempts to seek help or unsuccessful attempts to die. Generally, the act is non-habitual and may be influenced by single or multiple crises.

In this paper we illustrate epidemiological findings on attempted suicide in South Asian people in their countries of origin and in the UK and compare social and cultural factors across the two settings.

\section{The Indian subcontinent}

Most reported data have been collected from general hospitals. One of the key problems in identifying rates of attempted suicide is that of defining the act. A second problem is that, where suicide is legally proscribed, individuals or their families give different reasons for the action to hide the problems rather than admit to suicidal thoughts or acts.

In Madurai in South India, the number of patients presenting with attempted suicide trebled between 1974 and 1978. Of the 114 suicide attempts studied in 1965, 65 were by men and this male dominance persisted in data collected 2 years later. The dominant feelings of those attempting suicide (attempters) were depression, anger, spite, jealousy and a desire for attention. When those who repeated a suicide attempt (repeaters) were studied 10 years later, 19 out of 35 were male. The clinician described 20 as 'hysterical' with inadequate or immature personalities, eight had schizophrenia, three were dependent on drugs, two had a stammer, one had epilepsy and one had a toxic psychosis. The absence of depression was striking (Venkoba Rao \& Chinnian, 1972).

When differentiating between suicide and attempted suicide on the basis of psychological factors, Venkoba Rao (1992) cautions that suicide does not result from a single cause. He suggests that attempted suicide should be linked with personality disorders and argues that social isolation contributes to increased rates of suicide and attempted suicide.

Quarrels with in-laws and problems in interpersonal relationships appear to be extremely common causes of attempted suicide (Ponnudurai et al, 1986; Bannerjee et al, 1990). Repeat attempts are linked with alcohol misuse and poor personal relationships.

In a 2-year follow-up, Ponnudurai et al (1991) found that a sizeable minority of participants could not be traced at the address given. Of those who were traced, $14 \%$ continued to have suicidal intent and men were three times more likely to show

Dinesh Bhugra (Institute of Psychiatry, De Crespigny Park, London SE5 8AF, UK) is a reader in cultural psychiatry. His research interests include cultural factors in the genesis and management of psychiatric disorders and spirituality. Manisha Desai is a specialist registrar for Ealing, Hammersmith and Hounslow NHS Trust, London, with interests in gender, self-harm and mental illness. 
this than women. For women, their husbands' unemployment and alcohol misuse were relevant factors in suicidal thought and intent.

Ponnudurai et al (1986) had originally studied 86 patients in Madras and reported that in $10 \%$ of cases alcohol had played a significant role and that more than one-third of patients (38\%) had made a suicide attempt using organophosphorus compounds. Males were more likely to attempt suicide than were females, with those aged 15-20 years being the most vulnerable. This confirmed the findings of earlier studies (Venkoba Rao, 1965; Sathyavathi, 1971). Interpersonal difficulties and arguments had led to suicide attempts in $25 \%$ of patients. Unemployment and alcohol misuse were also important factors.

Khan \& Reza (1998), reporting from Karachi, found that women were more likely to attempt suicide than men and the causative factors included family conflicts, problems with their mothers-in-law and domestic violence.

In Sri Lanka, it has been suggested that an increase in the suicide rate could be linked to the easy access to poisonous agricultural chemicals (Ganesvaran et al, 1984). Marecek (1998) found that, when asked about suicide using a case vignette, a Sri Lankan sample suggested causes that included academic failure and interpersonal difficulties, especially disappointments in love. This sample attributed disturbed feelings and actions to planetary influences.

Mahla et al (1992) hypothesise that, in India, selfimmolation as a means of suicide is related both to psychiatric diagnosis and social factors. Illustrating their cases by linking them with a single sociopolitical event, they suggest that a breakdown of tradition may contribute to suicide rates.

From newspaper articles, Bhugra (1991) reported that politically motivated suicides were dramatic and carried out in public.

Kumar et al (1995) reported on 50 consecutive suicide attempts and found that $90 \%$ of patients had a psychiatric diagnosis and the risk of suicide was linked with this. These findings are not universal.

\section{South Asian migrant populations}

We now compare these rates with those reported among South Asian populations originating from the Indian subcontinent which have settled in other countries around the world.

Several studies have indicated that the rates of attempted and successful suicide are high among South Asians around the world.
In a study from a hill resort in Malaysia, where three-quarters of parasuicides were by Indians, most of whom were Tamils, Maniam (1988) hypothesised that the ambivalent attitudes of the Hindu religion towards suicide may account for part of the increased rates of suicide and attempted suicide. The study showed that younger Asian females had higher suicide and attempted-suicide rates and the most common method used was poisonous agricultural chemicals. Common precipitating factors included interpersonal difficulties and family conflicts, especially intercaste love problems.

Studying suicuide in Fiji, Haynes (1984) reported that rates of suicide among Indians were higher in older males and young women than in the native Fijian population. She argued that, because of stereotyping and their historical low status and because they were a political minority, underrepresented in government and the professional classes, the Indians were probably particularly vulnerable to attempted suicide.

Similar findings have been reported in Trinidad (Mahy, 1993). Studies there too have shown that interracial relationships and marriages play a very important role in precipitating suicide attempts. Paraquat was reported to be one of the key substances used by Trinidadian Asians.

\section{Adolescents in the UIK}

A different set of problems appears to lead to attempted suicide by adolescents in the UK. Diekstra (1985) proposed that an increase in the rates of suicide and suicide attempts among the young in Europe could be attributed to the interplay of three sets of factors: the socialisation of a specific problemsolving behaviour repertoire; socio-economic conditions; and attitudes towards suicide. Certainly, all these factors will be equally applicable to adolescents of Asian and other ethnic minority extraction.

Hawton et al (1982a) found that adolescents who take overdoses differ markedly in terms of factors concerning their families, especially their relationships with their parents, and in their medical and psychiatric histories, the nature of the overdoses and the problems that they were facing at the time. In a consecutive sample of 50 (White) adolescents admitted to hospital, 45 were girls (Hawton et al, 1982b). The commonest difficulties identified were problems in relationships with parents and boyfriends, or at school.

It is in schools that Goldberg \& Hodes (1992) highlighted the problems related to racism. They hypothesise that racism is bound to influence the family life cycle as well as the internal family organisation. An important issue is the view taken 
by the family and the adolescents of the severity of the external threat related to racism. The responses of each member of the family have to be understood in context. These authors propose that racism is an attack on the individual. In overdosing it is reproduced as an attack on self.

Several small retrospective studies of Asian adolescents in Coventry and Barnsley showed no differences in rates of attempted suicide when compared with White adolescents (Biswas, 1990; Handy et al, 1991).

Handy et al (1991) reported on adolescents who had taken overdoses and found that social and parental relationships were a key cause of isolation and attempted suicide. Asian adolescents were less likely to be in contact with their friends, saw them less frequently and for shorter periods, and their relationships with their parents did not compensate for this. Most Indian communities maintain their traditional cultural identity and uphold the importance placed on academic and economic success, the stigma attached to failure, the overriding authority of elders and an unquestioning compliance from the younger members.

\section{The UK}

As mentioned above, there have been very few studies of attempted suicide in ethnic minority groups in the UK. Of these, Burke's (1976) was a retrospective case note study and Merrill \& Owen's (1986) was a prospective study focusing on admissions in the same geographical area of the West Midlands. The first study from West London was by Bhugra et al (1999c), although previous audits had been reported from other areas of the city (e.g. Glover et al, 1989).

In a retrospective study of Birmingham's Asian immigrants, Burke (1976) observed that crude adjusted suicide rates were twice as high in females than in males, although repeat attempts were infrequent. Agricultural chemicals were rarely taken. Overall, the number of suicide attempts was low among Asians when compared with the base population. More than half (58\%) attributed their attempt to interpersonal problems, and a quarter gave no reason. The crude adjusted rates for the immigrants were in the middle of the range reported for natives in India and natives in Edinburgh.

In a prospective study from Birmingham nearly a decade later, Merrill \& Owens (1986) reported that the picture was beginning to change. All cases admitted to a hospital following attempted suicide were studied over a 2-year period. Of 196 Asian patients recruited, 139 were born on the Indian subcontinent, 52 were born in the UK and a few were born in East Africa. Females were almost three times as likely to present with attempted suicide and were of a younger age group.

Females of Asian origin reported marital problems significantly more frequently and many of these problems were due to cultural conflicts. Arranged marriages, rejection of arranged marriages and associated marital problems were reported as contributory factors by the Asian female sample. Since the researchers were White and male, they acknowledge that they may have missed some important and relevant information.

Merrill \& Owens (1988) studied attempted suicide in four immigrant groups (Asian, West Indian, Irish and Scottish) and in an English-born sample. The annual rates of self-poisoning in the immigrant groups exceeded rates in their countries of origin. Among the Asians and West Indians, males and older females were underrepresented but young females had similar rates to those of the Englishborn group. Interesting issues are raised by considering whether this is explained by Cochrane's selection hypothesis (Cochrane, 1977, 1983), that if migration is easy, less-stable individuals may selfselect to migrate. Furthermore, reasons for migration and associated stress may need to be studied under these circumstances to explain why suicide is attempted rather than how.

Glover et al (1989) analysed the records of patients aged 10-24 years presenting with self-poisoning to the two casualty departments at the London Hospital between 1980 and 1984. There were no reported suicides, but Asian females showed an excessive rate of attempted suicide compared with the base population. Wright et al (1981) also found high rates of attempted suicide in Birmingham.

As their inclusion criteria in a London sample, Bhugra et al (1999a) used Diekstra \& Gulbinat's (1993) definition of attempted suicide as:

\footnotetext{
'suicidal gestures and manipulative attempts to serious but unsuccessful attempts to kill oneself; any act with non-fatal outcome that attempts to cause or actually causes self-harm or, without intervention from others, would have done so, or consists of ingesting a substance in excess of its generally recognised or prescribed therapeutic dose'.

Habitual self-mutilation and alcohol misuse as primary presentations were not included. Efforts were made in the first instance to gauge as accurately as possible the inception rates of attempted suicide over a 1-year period. Details of the method are published elsewhere (Bhugra et al, $1999 b, c)$. The rates of attempted suicide among young South Asian women were reported to be higher than those for White females in the same age group.
} 


\section{Social and cultural factors}

As well as establishing the rates of attempted suicide, Bhugra et al (1999b) also studied various social and cultural factors. South Asian women who had attempted self-harm who agreed to be interviewed in detail about their cultural identity and life events were age-matched with an equal number of South Asian women attending a GP's surgery in the same catchment area and also with nearly twice the number of White women who had also presented with deliberate self-harm.

The comparison between the two Asian groups showed that those attempting suicide were significantly more likely to have history of past psychiatric disorder, more likely to repeat the attempt and more likely to be in interracial relationships. They were also more approving if their children wanted to have interracial relationships or choose their marriage partners themselves. Interestingly, one-quarter of each group acknowledged having experienced a racial life event in the previous 6 months. This is defined as any life event which the individual identifies as having a racial component, e.g. rejection of a mortgage loan application on racial rather than financial grounds. Thus, a racial life event per se is not likely to lead to self-harm. Furthermore, controls were more likely to see arranged marriage as good and were less likely to approve of cohabitation before marriage. Those attempting suicide were more likely to have changed their religion and spent less total time with their families.

When Asians attempting suicide were compared with White attempters, interesting differences emerged. Asian attempters were more likely to have no psychiatric disorder, were less likely to have used alcohol as part of the suicide attempt and were more likely to have been assaulted physically or verbally. The methods of attempted suicide for each group were broadly similar but Asians took fewer tablets. Suicidal intent and regret that their attempt was unsuccessful were more common in the Asian sample. The findings need to be interpreted with caution as only a small number of individuals were interviewed (Bhugra et al, 1999b).

Social and cultural factors that influence rates of self-harm in South Asian women are shown in Box 1.

In a case note study of Asian adolescents, Bhugra et al (2002) observed that inception rates for attempted suicide among teenagers were no different from those of their White counterparts; neither were the method used in the attempts nor the associated sociocultural factors. Nearly one-quarter of both Asian and White adolescents had conduct problems and a similar proportion had acknowledged a communication problem. Asian female adolescents were marginally more likely to report a family history
Box 1 Social and cultural factors influencing rates of self-harm in South Asian women

Gender: self-harm is more likely in females

Gender-role expectations

Alienation from culture, especially one's own but also from that of the majority population

Family conflict, e.g. with parents, partner

Domestic violence: by male members

Alcohol use in the family: by male members

Cultural conflict: liberal views $v$. traditional setting

Psychological distress expressed in the individual's alienation and rejection of cultural values

Poor self-esteem

of suicide and, remarkably, more likely to acknowledge cultural conflict. Asians were more likely to admit that their attempt was impulsive, were less likely to express regret after the attempt and were more likely to be at risk of attempts in the future.

White and Asian female adolescents (aged 16-17 years) had broadly similar adjustment reactions, alcohol and drug use, peer and relationship problems. However, the rates for Asian females aged 18-24 increased, suggesting that they came under more stress. This may relate to gender role expectations, individuation and culture conflict, which may contribute to attempts at self-harm. Thus, gender, gender-role expectations, family conflicts, domestic violence and alcohol use by males in the family may well contribute to stress and an increased propensity to attempt suicide. These need to be studied further.

\section{Assessing suicide risk in Asians}

General risk factors for suicide in any population are: past psychiatric history and present psychiatric symptoms such as depression, schizophrenia, alcohol dependency and psychopathic personality disorder; social factors such as social isolation and unemployment; physical illness; past history of suicide attempts; and labile mood.

Risk factors for suicide attempts include: recent attempts, especially if these were premeditated; precautions taken to avoid discovery and intervention by others; whether the attempt occurred in social isolation; prior communication of suicidal intent; the completion of final acts in anticipation of death, such as writing a will or a suicide note; violent methods; acts seen as lethal and irreversible; regrets 
Box 2 Assessing a recent suicide attempt

Was the act impulsive?

Was any action taken to prevent outsider intervention?

Was the risk of discovery minimised?

Had suicidal intent been communicated verbally or otherwise and to whom?

What was the chosen suicide method?

Did the individual believe that the act would be fatal?

Does the individual regret surviving the attempt?

Did the individual attempt to seek help after the act?

Does the individual have a history of previous attempts? Ascertain details of the act

on surviving the attempt; and taking no action to get help after the event. Formation of a suicide plan, an inclination to deal with internal conflict and recent experience of failure and rejection are also important factors. Bhugra et al (1999b) found that Asian women were more likely to repeat overdoses, which suggests that adequate preventive strategies may be successful. As a significant proportion of attempted suicides by Asian females are impulsive, any assessment and intervention should also take impulsive acts into account.

The process of assessing suicide risk in Asians is basically the same as that for other groups (Box 2). However, additional information, especially on social and cultural alienation, social support, family structures and expectations as well as such factors as less-traditional thinking, must be explored as a key part of the assessment (Box 3).

It is important that clinicians determine precipitating events, motives for the act, consequences of

\section{Box 3 Additional factors for assessing Asians}

Assess the following:

Migration (if indicated) and associated stressors and social support

Cultural identity and cultural alienation

Gender-role expectations

Type of family network, e.g. extended, nuclear Social support and confidants

Cultural idioms of distress and expectations Any prior racial life events

Note that suicidal motivation may be interpreted by the attempter in terms of cultural expectations of behaviour the act, precautions taken against discovery, preparatory acts and symptoms of depression as well as the family's reactions and attitudes, and that an examination of the patient's physical and mental state is carried out as indicated.

Clinicians assessing deliberate self-harm should use non-directive, open questions and appropriate language. They should also take into account the cultural values and beliefs of the individual, including their religious views. If interpreters are needed, these should be independent rather than family members.

\section{Conclusions}

An individual's ethnicity and cultural group remain useful starting points for understanding the motives behind attempted suicide as well as for assessing the probability that the attempt will be repeated. The impact of migration and socio-economic and political disadvantages may well contribute to a sense of chronic ongoing difficulty and act as potential stressors. When these are combined with a sense of alienation from their own culture, this may well reduce people's sense of belonging and acceptance and influence their self-esteem, which may produce an affective reaction. It is also possible in society-centred cultures and groups that if the density of individuals from the same ethnic group is great, there will be an inordinate pressure on individuals to conform. Under the circumstances, any vulnerable individual in conflict with their own culture may choose to take the route of self-harm to avoid dealing with that conflict.

Clinicians must be aware of the differences between those who consider suicide, those who attempt it and those who are successful. The association between these three groups and their relationship with other factors needs to be further clarified. Suicidal behaviour needs to be studied across the life cycle in relation to gender roles and cultural and social expectations. Definitions of the self also differ and are influenced by culture and society. Hence, it becomes important that the individuals' perceptions of self are identified in the context of their culture.

\section{References}

Bannerjee, G., Nandi, D., Nandi, S., et al (1990) The vulnerability of Indian women to suicide. Indian Journal of Psychiatry, 32, 305-308.

Bhugra, D. (1991) Politically motivated suicides. British Journal of Psychiatry, 159, 594-595.

—, Desai, M. \& Baldwin, D. (1999a) Attempted suicide in 
West London. I: Inception rates. Psychiatric Medicine, 29, 1125-1130.

—, Baldwin, D., Desai, M., et al (1999b) Attempted suicide in West London. II: Social and cultural factors. Psychiatric Medicine, 29, 1131-1139.

—, Bhui, K. S., Desai, M., et al (1999c) The Asian Cultural Identity Schedules: an investigation of culture and self harm. International Journal of Methods in Psychiatric Research, 8, 212-218.

-, Singh, J., Bayliss, C., et al (2002) Attempted suicide in adolescents - a case note study. European Journal of Psychiatry, in press.

Biswas, S. (1990) Ethnic differences in self-poisoning. Journal of Adolescence, 13, 189-193.

Burke, A. W. (1976) Attempted suicide among Asian immigrants in Birmingham. British Journal of Psychiatry, $128,528-533$.

Cochrane, R. (1977) Mental illness in immigrants to England and Wales. Social Psychiatry, 12, 25-35.

- (1983) The Social Creation of Mental Illness. London: Longman.

Diekstra, R. F. (1985) Suicide and suicide attempts in the European Economic Community: an analysis of trends, with special emphasis upon trends among the young. Suicide and Life Threatening Behaviour, 15, 27-42.

— \& Gulbinat ,W. (1993) The epidemiology of suicidal behaviour: a review of three continents. World Health Statistics Quarterly, 46, 52-68.

Glover, G., Marks, F. \& Nowers, M. (1989) Parasuicide in young Asian women. British Journal of Psychiatry, 154, 271-272.

Goldberg, D. \& Hodes, M. (1992) The poison of racism and the self poisoning of adolescents. Journal of Family Therapy, 14, 51-67.

Gunesvaran, T., Subramaniam, S. \& Mahadeva, N. K. (1984) Suicide in a northern town in Sri Lanka. Acta Psychiatrica Scandinavica, 69, 420-425.

Handy, S., Chithiramohan, R., Vallard, C., et al (1991) Ethnic differences in adolescent self poisoning: a comparison of Asian and Caucasian groups. Journal of Adolescence, 14, 157-162.

Hawton, K., Osborn, M., O'Grady, J., et al (1982a) Classification of adolescents who take overdoses. British Journal of Psychiatry, 140, 124-131.

-, O'Grady, J., Osborn, M., et al (1982b) Adolescents who take overdoses: their characteristics, problems and contacts with helping agencies. British Journal of Psychiatry, 140, 118-123.

Haynes, R. H. (1984) Suicide in Fiji: a preliminary study. British Journal of Psychiatry, 145, 433-438.

- (1987) Suicide and social response in Fiji: a historical survey. British Journal of Psychiatry, 151, 21-26.

Khan, M. M. \& Reza, H. (1998) Attempted suicide in Karachi, Pakistan. Suicide and Life Threatening Behaviour, 28, 54-60.

Kumar, P. N. S., Kuruvilla, K., Dutta, S., et al (1995) Psychosocial aspects of attempted suicide: study from a medical intensive care unit. Indian Journal of Psychological Medicine, $18,32-42$.

Mahv, G. (1993) Suicide behaviour in the Caribbean International Review of Psychiatry, 5, 261-269.

Marecek, J. (1998) Culture, gender and suicidal behaviour in Sri Lanka. Suicide and Life Threatening Behaviour, 28, 69-81.

Merrill, J. \& Owens, J. (1986) Ethnic differences in selfpoisoning. British Journal of Psychiatry, 148, 708-712.

— \& Owens, J. (1988) Self-poisoning among four immigrant groups. Acta Psychiatrica Scandinavica, 77, 77-80.

Nazroo, J. (1997) Ethnicity and Mental Health. London: PSI.

Ponnudurai, R., Jeyakar, J. \& Saraswathy, M. (1986) Attempted suicide in Madras. Indian Journal of Psychiatry, 28, 59-62.

-, Vivekanathan, V., Raju, B., et al (1991) Attempted suicide: two year follow up study. Indian Journal of Psychiatry, 33, 291-292.

Venkoba Rao, A. V. (1965) Attempted suicide. Indian Journal of Psychiatry, 7, 253-259.

- \& Chennian, R. (1972) Attempted suicide and suicide in students in Madurai. Indian Journal of Psychiatry, 14, 389-396.

Rao, A .V. (1992) Parasuicide and suicide: some psychological considerations. Indian Journal of Social Psychiatry, 8, 3-7.
Sathyavathi, K. (1971) Attempted suicide in psychiatric patients. Indian Journal of Psychiatry, 13, 37-42.

Somasundaram, O. S., Babu, C. K. \& Geelthayan, I. A. (1989) Suicide behaviour in ancient civilization with special reference to the families. Indian Journal of Psychiatry, 31, 208-212.

Wright, N., Trethowan, W. \& Owens, J. (1981) Ethnic differences in self poisoning. Postgraduate Medical Journal, 57, 792-793.

\section{Multiple choice questions}

1. Rates of attempted suicide in the Indian 'diaspora':
a are high
$\mathrm{b}$ are related to culture conflict
c can be related to low social status
$\mathrm{d}$ are never related to alcohol misuse
e may have religious causation in some cases.

2. Attempted suicide rates for South Asian women: a who are young are higher than for White females

$\mathrm{b}$ are not higher in teenagers

c are related to culture conflict

d are likely to be related to impulsive acts

e are higher among migrants.

3. South Asian suicide attempters are more likely to:

a be female

$b$ be repeaters

c have past psychiatric history

$\mathrm{d}$ be in interracial relationships

e have experienced racial life events.

4. Asian attempters are less likely than Whites to:

a have no psychiatric disorder

b have used alcohol in the attempt

c have a history of assaults

d take a large number of tablets

e experience regret after the attempt.

5. Adolescent Asian attempters are more likely than Whites to:

a report culture conflict

b report family history of suicide

c use alcohol

$\mathrm{d}$ be impulsive

e have conduct disorder.

\section{MCQ answers}

$\begin{array}{llllllllll}\mathbf{1} & & \text { 2 } & & 3 & & 4 & & 5 & \\ \text { a } & \text { T } & \text { a } & \text { T } & \text { a } & \text { T } & \text { a } & \text { F } & \text { a } & \text { T } \\ \text { b } & \text { T } & \text { b } & \text { F } & \text { b } & \text { T } & \text { b } & \text { T } & \text { b } & \text { T } \\ \text { c } & \text { T } & \text { c } & \text { T } & \text { c } & \text { T } & \text { c } & \text { F } & \text { c } & \text { F } \\ \text { d } & \text { F } & \text { d } & \text { T } & \text { d } & \text { T } & \text { d } & \text { F } & \text { d } & T \\ \text { e } & \text { T } & \text { e } & \text { T } & \text { e } & \text { F } & \text { e } & \text { F } & \text { e } & \text { F }\end{array}$

